\title{
A THEORY OF REFERENCE-DEPENDENT BEHAVIOR
}

\author{
JOSE APESTEGUIA ${ }^{\dagger}$ AND MIGUEL A. BALLESTER ${ }^{\ddagger}$
}

\begin{abstract}
Extensive field and experimental evidence in a variety of environments show that behavior depends on a reference point. This paper provides an axiomatic characterization of this dependence. We proceed by imposing gradually more structure on both choice correspondences and preference relations, requiring increasingly higher levels of rationality, and freeing the decision-maker from certain types of inconsistencies. The appropriate degree of behavioral structure will depend on the phenomenon that is to be modeled. Lastly, we provide two applications of our work: one to model the status-quo bias, and another to model addictive behavior.
\end{abstract}

Keywords: Individual rationality, reference-dependence, rationalization, path independence, status-quo bias, addiction, habit formation.

JEL classification numbers: A12, B41, D11.

\section{INTRODUCTION}

Extensive evidence has accumulated over the last few decades to suggest that behavior in a variety of settings is in part determined by a reference point. The reference point may be interpreted as the default choice, as in the status quo bias or endowment effect literature; the aspiration level, as in aspiration adaptation models; the convention, norm, or belief about what one should choose, as, for example, in cognitive dissonance studies; past consumption, as in addiction, habit formation, status-seeking, or brand loyalty models; and others.

This paper provides a conceptual framework for the systematic study of referencedependent behavior. The approach we follow is axiomatic and gradual. We study the properties of reference-dependent choice correspondences and reference-dependent preferences that impose gradually more structure on both choice correspondences and preference relations, requiring increasingly higher levels of rationality, and freeing the decision-maker from certain types of inconsistencies. The appropriate degree of behavioral structure will depend on the phenomenon that is to be modeled. We focus on those cases where the reference point, if it exists, is itself an element of the choice

Date: September, 2007.

We thank the participants of many seminars and conferences for their valuable comments. Special thanks are due to a referee for many comments, suggestions and criticisms that have led to a substantial improvement in the manuscript. The research was sponsored by the Spanish Commission of Science and Technology (SEJ2006-11510) and the Barcelona Economics Program of CREA.

$\ddagger$ Universitat Pompeu Fabra. E-mail: jose.apesteguia@upf.edu.

‡Universitat Autonoma de Barcelona. E-mail: miguelangel.ballester@uab.es. 
set. The reference point, moreover, changes with each choice, in the sense that the last choice is the new reference point.

The gradual incorporation of behavioral structure described in this paper involves four steps. In the first, we study the properties that are needed to rationalize reference-dependent choice correspondences. That is, we explore the conditions that a choice correspondence needs to satisfy in order to be explained by a book of preference relations. ${ }^{1}$ We argue that this consistency requirement is a minimal rationality condition to be imposed on a formal treatment of reference-dependent behavior. By extending properties $\alpha, \beta$, and the weak axiom of revealed preference to choice problems with reference points, we obtain that a choice correspondence satisfies properties $\alpha$ and $\beta$, if and only if, it satisfies the weak axiom, if and only if, there exists a book of complete preorder relations that rationalizes the choice correspondence. Interestingly, we show that this book may be incomplete (and even intransitive) in the space of alternatives lying "below" the reference point. Further, we show that the book is unique in the behaviorally relevant space, that is in all those pairs that are "above" the reference point.

We then study non-dominated behavior. By non-dominated behavior we mean situations where there are no cycles. For example, let an agent be confronted with a choice problem involving two alternatives, $x$ and $y$, and let us assume that when she chooses from reference point $x$ she strictly prefers $y$ and vice versa. If the last choice determines the next reference point, the agent will repeatedly switch back and forth between $x$ and $y$. Clearly, such erratic behavior is not consistent even with minimal rationality requirements. We identify the axioms on preferences and on choice correspondences that guarantee non-dominated behavior.

Furthermore, we say that behavior is independent of the initial reference point if, given a choice set, final choices (to be formally defined below) cannot be influenced by the initial choice of reference point. Our next result characterizes the typology of choice correspondences and books of preferences that satisfy independence of the initial reference point.

Finally, a natural step further in the study of the rationality of reference-dependent behavior is the analysis of when behavior is not only independent of the initial reference point in a given choice set, but also independent of the way in which the choice problem is presented. That is, we will say that behavior is path-independent whenever final choices do not depend on the sequence in which choice problems are presented.

After imposing all this structure, the immediate question that arises is whether behavior is still reference-dependent, or collapses instead into a unique order, as in the classical setup. We will show by means of an example that there is still room for reference-point dependence.

It is immediate that the classic behavioral case, where there is a unique complete preorder rationalizing a single choice correspondence satisfying the classic version of the weak axiom of revealed preference, is free from all these inconsistencies. That

\footnotetext{
${ }^{1}$ Books of preference relations will be precisely defined below. For now, consider a book of preference relations as a collection of preference relations, one for each of the elements in the universal set of alternatives, and one that represents the preferences when there is no reference point.
} 
is, it shows non-dominated behavior that is independent of starting conditions and also path-independent. Our analysis, then, evaluates the degree of rationality of reference-dependent behavior against the classic case. This seems to us to be a natural starting point for a deep theoretical understanding of reference dependent behavior. Furthermore, our framework suggests a measure of the degree of rationality of reference-dependent behavior. It may be argued that the severity of a particular reference-dependent phenomenon may be estimated from the place it takes in the gradual structure we propose.

We end the paper by applying the framework derived here to two empirically wellestablished; status quo bias and addiction. We show that, although the characterizations differ in each case, choice behavior, be it subject to the status quo bias or to addiction, can be rationalized by a book of complete preorders that satisfies nondominated behavior, without necessarily being independent of initial reference points or path-independent.

The paper is organized as follows. Section 2 gives a brief review of related literature. Section 3 introduces the notation that will be used subsequently. Section 4 contains the analysis of the four structural steps that may be imposed to reference-dependent behavior. In section 5 we develop two applications. Section 6 presents the conclusions. All the proofs are given in section 7 .

\section{Related Literature}

To the best of our knowledge, Tversky and Kahneman (1991) were the first to provide an explicit model of reference-dependent preferences. Their model is an extension of Kahneman and Tversky's (1979) prospect theory to riskless choices, designed to model the status quo bias and related issues. They work with a book of reference-dependent preferences on a two-dimensional commodity space, and assume, instead of axiomatically deriving, certain types of behavior (such as loss aversion) that lead to the status quo bias.

Munro and Sugden (2003) revise and extend Tversky and Kahneman's (1991) model to the $n$-dimensional commodity space. Sagi (2006), Sugden (2003), Giraud (2004), and Köszegi and Rabin (2006) constitute further developments. Sagi (2006) studies the structure of the book of preference relations at the light of imposing a "no-regret" consistency property (see section 4.2 below for an elaboration on this property), both in riskless and risky settings. Sugden proposes a generalization of Savage's expected utility theory to the case where preferences are reference-dependent. Giraud deals, among other things, with the elusive question of the welfare evaluation of referencedependent behavior. These three papers have in common that they are axiomatic, and that they study the relationship between different reference-dependent preferences. Finally, Köszegi and Rabin study the case where the reference point is determined by decision-maker outcome expectations. ${ }^{2}$

One strand of the literature follows the revealed preference approach pioneered by Zhou (1997). Zhou provides an axiomatic foundation for the status quo bias for

\footnotetext{
${ }^{2}$ See also the work of Bleichrodt $(2006,2007)$ and Giraud $(2006,2007)$.
} 
choice functions. His approach has been followed by Bossert and Sprumont (2006), and by Masatlioglu and Ok (2005). Bossert and Sprumont consider status quo choice correspondences that select all those alternatives that are at least as good as the status quo. The closest paper to ours is Masatlioglu and Ok (2005). They provide a powerful characterization of the status quo bias. In section 5.1, we derive, on the preferences side, the properties that are equivalent to theirs. Furthermore, their framework includes the study of choice problems with and without reference points, which is the view we adopt here. ${ }^{3}$

The contributions of our analysis to the literature are two-fold. First, we axiomatically study reference-dependent behavior from the perspective of both choice correspondences and preferences. Second, we provide a general axiomatic baseline in a riskless setting that starts by imposing minimal rationality assumptions, and then gradually incorporates more structure into reference-dependent behavior, thus freeing the decision-maker from certain types of inconsistencies.

\section{Notation}

Let $X$ be an arbitrary nonempty set of elements, representing the space of alternatives, and $\mathcal{X}$ the set of all nonempty subsets of $X$. For notational convenience, and following Masatlioglu and Ok (2005), we use $\diamond$ to denote an element such that $\diamond \notin X$. It will be convenient to define $X^{*}=X \cup\{\diamond\}$. A choice problem is a pair $(T, s)$, where $T \in \mathcal{X}$, and either $s \in T$ or $s=\diamond$. When $s \in T$ we say that $(T, s)$ is a choice problem that depends on a reference point, and when $s=\diamond$ we say that $(T, s)$ is a choice problem that does not depend on a reference point. $\mathcal{C}(X)$ represents the set of all possible choice problems $(T, s)$, while $\mathcal{C}_{\text {rd }}(X)$ denotes the set of all possible choice problems with a reference point.

A choice correspondence $c(T, s)$ assigns for every $(T, s)$ a subset of $T$ in $\mathcal{X}$. Hence, $c: \mathcal{C}(X) \rightarrow \mathcal{X}$, where $c(T, s) \subseteq T$ for all $(T, s) \in \mathcal{C}(X)$. Note that, as usual, $c$ is by definition a non-empty valued mapping.

We now introduce reference-dependent preference relations. For every $s \in X^{*}$ denote by $\succeq_{s}$ a binary relation on $X, \succeq_{s} \subseteq X \times X$. We denote by $\left\{\succeq_{s}\right\}_{s \in X^{*}}$ the collection of all such binary relations. We refer to this collection as the book of reference-dependent binary relations associated to $X^{*}$.

Binary relations $\succ_{s}$ and $\sim_{s}$ are the asymmetric and symmetric parts of $\succeq_{s}$, respectively. Hence, $x \succ_{s} y$ if, and only if, $x \succeq_{s} y$ and $\neg\left(y \succeq_{s} x\right)$, and $x \sim_{s} y$ if, and only if, $x \succeq_{s} y$ and $y \succeq_{s} x$. We will say that a binary relation is a preorder if it is reflexive and transitive, and a partial order if it is an antisymmetric preorder. For any $(T, s), M\left(T, \succeq_{s}\right)$ denotes the set of maximal elements in $T$ with respect to $\succeq_{s}$, that is, $M\left(T, \succeq_{s}\right)=\left\{x \in T: y \succ_{s} x\right.$ for no $\left.y \in T\right\}$, and $G\left(T, \succeq_{s}\right)$ denotes the set of greatest elements in $T$ with respect to $\succeq_{s} ; G\left(T, \succeq_{s}\right)=\left\{x \in T: x \succeq_{s} y\right.$ for every $\left.y \in T\right\}$. Finally, the restriction of a reference-dependent preference relation $\succeq_{s}$ to any subset

\footnotetext{
${ }^{3}$ Masatlioglu and Ok (2006) and Masatlioglu and Uler (2006) constitute recent developments in the revealed preference approach.
} 
$T \subseteq X$ is the relation $\left.\succeq_{s}\right|_{T}$ on $T$ such that, for all $x, y \in T, x \succeq_{s} y$ if and only if $\left.x \succeq_{s}\right|_{T} y$.

\section{Reference-Dependent Behavior}

In this section we introduce four steps that gradually incorporate more structure into reference-dependent behavior. The "adequate" degree of behavioral structure will be determined by the phenomenon that is to be studied.

In section 4.1 the problem of rationalization of reference-dependent behavior is studied. There, rationality requirements are imposed given a reference point. However, the absence of any connection among the different orderings that conform an individual book of preferences may be the origin of inconsistencies in individual behavior. In sections $4.2,4.3$, and 4.4 we study this type of inconsistency using a very simple model of nested reference points. We assume that the reference point corresponds to the default option of the decision-maker and second, the last choice defines the next reference point. That is, we do not initially presume complex rationality in the behavior of our agent. She takes current decisions according to the preference ordering related to her default option, without anticipating how this will influence future decisions. Of course we could alternatively assume that the decision-maker anticipates how actual choices will determine subsequent reference points, and hence future choices. However, in this paper we choose the above explained simple myopic version as the starting point, and only obtain sophisticated reference-dependent behavior as the result of certain rationality principles we will impose (see sections 4.3 and 4.4 below).

Now consider the following definition.

Definition 4.1. Let $\left(T_{i}, s_{i}\right)_{i=0}^{n}$ be a sequence of reference-dependent choice problems and let $c$ be a choice correspondence. Then $\left(T_{i}, s_{i}\right)_{i=0}^{n}$ is an RD-chain for $c$ if $s_{i} \in T_{i}, T_{i}=T_{j}=T$ for all $0 \leq i, j \leq n$ and $s_{i} \in c\left(T, s_{i-1}\right)$ for all $i=1,2, \ldots, n$. An RD-chain is cyclical if $s_{0} \in c\left(T, s_{n}\right)$. An RD-chain is strict if there exists $k$ in $\{0,1, \ldots, n-1\}$ such that $s_{k} \notin c\left(T, s_{k}\right)$.

Note that the above defines RD-chains on the basis of a single, fixed choice set $T$. Three examples are introduced below. The first gives an instance of strict and cyclical RD-chains, the second, one of cyclical but non strict RD-chains, and the third, one of strict and non cyclical RD-chains.

Example 4.2. Let $X=\{x, y\},\{x\}=c(X, y),\{y\}=c(X, x)$, and $X=c(X, \diamond)$.

Example 4.3. Let $X=\{x, y\},\{y\}=c(X, y),\{x\}=c(X, x)$, and $X=c(X, \diamond)$.

Example 4.4. Consider the set $X=\mathbb{N}$ where $\mathbb{N}$ is the set of natural numbers, and the correspondence $f: X \longrightarrow \mathcal{R}$ defined as follows:

$$
f(x)=\left[\frac{x}{k}, k x\right],
$$


where $k>2$. Take a choice correspondence such that for any $(T, s) \in \mathcal{C}_{\mathrm{rd}}(X)$, it is $c(T, s)=z$ where $z$ is the greatest element in the set $T \cap f(s)$. Finally, for any $(T, \diamond)$, let $c(T, \diamond)=T$.

The interpretation of examples 4.2 and 4.3 is straightforward. Note that in example 4.2 , starting at $(X, x)$, the agent goes to $(X, y)$, and then back to $(X, x)$. This clearly constitutes a cyclical and strict RD-chain. In example 4.3, starting, e.g., from $(X, x)$, goes to $(X, x)$, representing a cyclical non-strict $\mathrm{RD}$-chain. The behavior described in example 4.4 corresponds to an agent who, when endowed with a reference point $s \in X$ maximizes the consumption of a "good" in a neighborhood of its reference point $s \in \mathbb{N}$. For example, take $T=X, s=1$ and $k=3$. It is easy to see that from $(X, 1)$, we move to $(X, 3)$, and then to $(X, 9),(X, 27)$, and so on, which illustrates the case of a non-cyclical and strict RD-chain.

Note that in Definition 4.1 it is stated that $s_{i} \in T$, hence one may wonder about RD-chains with $s_{i}=\diamond$. Note, however, that since $c(T, \cdot) \subseteq T$ then $s_{i}=\diamond$ is only possible when $i=0$, but then, for our purposes, one can take the RD-chain following $s_{0}=\diamond$.

4.1. Rationalization of Reference-Dependent Choice Correspondences. The question of the rationalization of reference-dependent choice correspondences addresses the problem of the existence of a book of binary relations that is consistent with choice behavior. Formally, a choice correspondence $c$ on $\mathcal{C}(X)$ is $M$-rationalized by a book of preorders $\left\{\succeq_{s}\right\}_{s \in X^{*}}$ if for any choice problem $(T, s) \in \mathcal{C}(X), c(T, s)=$ $M\left(T, \succeq_{s}\right)$. We will also be interested in a related rationalization notion, defined on the grounds of greatest elements: a choice correspondence $c$ on $\mathcal{C}(X)$ is $G$ rationalized by a book of preorders $\left\{\succeq_{s}\right\}_{s \in X^{*}}$ if for any choice problem $(T, s) \in \mathcal{C}(X)$, $c(T, s)=G\left(T, \succeq_{s}\right)$. Clearly, if the book is composed of complete preorders, then both notions coincide. Otherwise, note that $G\left(T, \succeq_{s}\right) \subseteq M\left(T, \succeq_{s}\right)$.

In a classic article, Arrow (1959) showed that in the context of choice problems without a reference point, Samuelson's weak axiom of revealed preference (WARP) guarantees that there is a unique complete preorder relation $\succeq_{\diamond}$ that rationalizes $c(\cdot, \diamond)$. Based on the version of Mas-Colell, Whinston, and Green (1995), we now present a variant of the weak axiom within the context of choice problems with a reference point.

Weak Axiom of Revealed Preference (WARP): For any $(T, s) \in \mathcal{C}(X)$ with $x, y \in T$ and $x \in c(T, s)$, then for any $(V, s) \in \mathcal{C}(X)$ with $x, y \in V$ and $y \in c(V, s)$, it must be that $x \in c(V, s)$.

It is well-known that, in the standard context, the weak axiom is equivalent to Sen's (1969) properties $\alpha$ and $\beta$. Consider the following adaptation of properties $\alpha$ and $\beta$, due to Masatlioglu and Ok (2005). 
Property $\alpha$ : For any $(T, s),(V, s) \in \mathcal{C}(X)$, if $y \in V \subseteq T$ and $y \in c(T, s)$, then $y \in c(V, s)$.

Property $\beta$ : For any $(T, s) \in \mathcal{C}(X)$, if $z, y \in c(T, s), T \subseteq V$, and $z \in c(V, s)$, then $y \in c(V, s)$.

As already informally mentioned in Masatlioglu and Ok (2005), the versions of properties $\alpha$ and $\beta$ for reference-dependent contexts are equivalent to the version of WARP introduced above. It turns out that properties $\alpha$ and $\beta$ (and consequently WARP) imply the existence of a book of preorders that rationalizes $c$. Furthermore, we show that the existence of such a book of preorder relations implies that the choice correspondence $c$ satisfies those variants of properties $\alpha$ and $\beta$.

The main difference between our setting and the classic one lies in the fact that, here, each choice correspondence $c(\cdot, s)$ is not defined on the whole $\mathcal{X}$, but only on the filter $\mathcal{X}_{s}:=\{S \in \mathcal{X}: s \in S\} . \mathcal{X}_{s}$ has the feature of not containing all pairs of objects. In particular $\{x, y\} \notin \mathcal{X}_{s}$ whenever $x \neq s$ and $y \neq s$. As a consequence of this, reference-dependent choice behavior is intimately connected to incomplete preference orders. ${ }^{4}$ That is, when there is a reference point $s \in X$, all that is required is to impose completeness over the set of alternatives that is no worse than the reference point $s$. No structure is needed "below" the reference point, since these elements are never chosen. Hence the book of preference relations may be incomplete, in this very specific way. ${ }^{5}$ The following result formalizes this discussion.

Lemma 4.5. For any choice correspondence $c$ on $\mathcal{C}(X)$, the following three statements are equivalent:

- c satisfies axiom WARP.

- $c$ satisfies properties $\alpha$ and $\beta$.

- There exists a book $\left\{\succeq_{s}\right\}_{s \in X^{*}}$ of preorders $\psi$-rationalizing $(\psi \in\{G, M\})$ such that for all $s \in X, \succeq_{s}$ is complete on the set $X_{s}:=\{x \in X: x \in c(\{x, s\}, s)\}$. Moreover, if $\left\{\succeq_{s}^{1}\right\}_{s \in X^{*}}$ and $\left\{\succeq_{s}^{2}\right\}_{s \in X^{*}}$ are two books $\psi$-rationalizing $c$, then $\left.\succeq_{s}^{1}\right|_{U_{s}}=\left.\succeq_{s}^{2}\right|_{U_{s}}$ for all $s \in X^{*}$, where $U_{s}=\left\{x \in X^{*}: x \succeq_{s} s\right\}$.

Lemma 4.5 shows that: (i) The book $\left\{\succeq_{s}\right\}_{s \in X^{*}}$ rationalizing $c$ can be formed by incomplete relations, but these must be complete for all elements above the reference point; (ii) The kind of rationalization, $G$ or $M$, is not fundamental, in the sense that a book $\left\{\succeq_{s}\right\}_{s \in X^{*}} G$-rationalizes $c$ if, and only if, $M$-rationalizes $c$. We may therefore speak simply of the rationalization of a choice correspondence $c$, forgoing the need

\footnotetext{
${ }^{4}$ Bleichrodt $(2006,2007)$ also deals with incomplete reference-dependent preferences in this sense.

${ }^{5}$ In fact, transitivity may also be relaxed accordingly. That is, transitivity is only required in the space "above" the reference point and it should be the case that all the alternatives "below" the reference point are strictly preferred by all the alternatives "above" the reference point. Then, violations of transitivity involving elements below the reference point are admissible. For ease of exposition, however, we assume throughout that the reference-dependent preferences are transitive.
} 
for the qualifier $\psi \in\{G, M\}$; (iii) The book of relations $\left\{\succeq_{s}\right\}_{s \in X^{*}}$ rationalizing $c$ is unique in the relevant behavioral part, that is in all those pairs lying above the reference points. In particular, for a given book $\left\{\succeq_{s}\right\}_{s \in X^{*}}$ defining the relation $\succeq$ on $X$ by $x \succeq y \Leftrightarrow x \succeq_{y} y$, if $\left\{\succeq_{s}^{1}\right\}_{s \in X^{*}}$ and $\left\{\succeq_{s}^{2}\right\}_{s \in X^{*}}$ are two books rationalizing $c$, then $\succeq^{1}=\succeq^{2}$.

Now, it is immediate that it is possible to complete the binary relations of the book $\left\{\succeq_{s}\right\}_{s \in X^{*}}$ that rationalizes $c$ to convert it into a complete preorder book. For example, for every $\succeq_{s}$ whenever $x, y \in X \backslash X_{s}$ write $x \sim_{s} y$. This is the completion that is performed in the following corollary.

Corollary 4.6. For any choice correspondence $c$ on $\mathcal{C}(X)$, the following three statements are equivalent:

- c satisfies axiom WARP.

- $c$ satisfies properties $\alpha$ and $\beta$.

- There exists a book $\left\{\succeq_{s}\right\}_{s \in X^{*}}$ of complete preorders rationalizing c.

Moreover, if $\left\{\succeq_{s}^{1}\right\}_{s \in X^{*}}$ and $\left\{\succeq_{s}^{2}\right\}_{s \in X^{*}}$ are two books rationalizing $c$, then $\left.\succeq_{s}^{1}\right|_{U_{s}}=\succeq_{s}^{2}$ $\left.\right|_{U_{s}}$ for all $s \in X^{*}$, where $U_{s}=\left\{x \in X^{*}: x \succeq_{s} s\right\}$.

Lemma 4.5 and Corollary 4.6 establish the baseline for an axiomatic treatment of reference-dependent behavior. In what follows and for ease of exposition, we will refer to books of complete preorders rationalizing $c$. Note, however, that all that is in fact needed is that the preorders $\succeq_{s}$ be complete in $X_{s}$ only. We now turn to the study of non-dominated behavior, independence of initial reference points, and path-independence.

4.2. Non-dominated Behavior. Non-dominated behavior is often imposed as one of the very first rationality requirements. For its motivation, consider the following "money-pump" argument. Take, in the context of the classical formulation: $x \succ y \succ$ $x$. Suppose the agent starts with option $x$. Since $y \succ x$, the agent could pay a small enough sum to obtain $y$ instead of $x$. After this trade, the agent could, by similar reasoning return to $x$. The agent comes back to the original element, but with her money endowment diminished, and would again be willing, for a small enough sum, to trade $x$ for $y{ }^{6}$

The latter is a clear example of the possible irrationality of intransitive agents, who can be manipulated into sequences of choices. The literature on reference-dependent preferences has played close attention to this sort of questions. Munro and Sugden (2002), Sugden (2003), Sagi (2006) and Giraud (2007) study the consequences of different kinds of restrictions.

\footnotetext{
${ }^{6}$ There are however many discussions on the general validity of the money-pump argument (see for example Raiffa 1968, Fishburn 1988, or Mandler 2005). In particular, it could be argued that, for the money-pump argument to be valid, preferences should be defined over the Cartesian product of money and alternatives. See Danan (2005) for a treatment in this direction.
} 
We start the analysis by considering a property proposed by Giraud (2007) that applies to pairs of alternatives only.

Status Quo (SQ): For any $x, y \in X, x \succeq_{y} y \Rightarrow x \succeq_{x} y$.

Clearly, SQ directly eliminates the money-pump argument in the case of two alternatives. Interestingly, in the presence of rationalizability, it is not difficult to show that $\mathrm{SQ}$ is equivalent to a property that plays an important role in the subsequent analysis:

Weak-NSC: When the opportunity set has two elements, there is no RD-chain associated to the choice correspondence $c$ that is cyclical and strict at the same time.

The proof is straightforward. It suffices to note that, according to Lemma 4.5, $x \succeq_{y} y$ and $y \succ_{x} x$ is equivalent to $x \in c(\{x, y\}, y)$ and $y=c(\{x, y\}, x)$. Thus, a violation of SQ is directly connected to a violation of Weak-NSC. The following result is therefore obtained.

Theorem 4.7. For any choice correspondence $c$ on $\mathcal{C}(X)$, the following two statements are equivalent:

- $c$ satisfies properties $\alpha, \beta$ and Weak-NSC.

- There exists a book $\left\{\succeq_{s}\right\}_{s \in X^{*}}$ of complete preorder relations that rationalizes $c$, and that satisfies $S Q$.

A natural extension of SQ to any number of alternatives is Weakly ExchangeAversion, which appears in Sugden (2003):

Weakly Exchange-Aversion (WEA): For any $s_{0}, s_{1}, \ldots, s_{n} \in X$ such that $s_{n} \succeq_{s_{n-1}} s_{n-1} \succeq_{s_{n-2}} s_{n-2} \ldots s_{1} \succeq_{s_{0}} s_{0}$, it must be $s_{n} \succeq_{s_{n}} s_{0}$.

The choice domain property can be extended similarly.

Non-Strict Cycles (NSC): There is no RD-chain associated to the choice correspondence $c$ that is cyclical and strict at the same time.

Note that the previous definition involves only choice problems with the same set of available options. Houthakker (1950) already proposed a property, "semitransitivity", that restricts the relation between the first and last elements of a chain. In his version, however, the choice set may change from one link to the other. Our consideration of a single set, then, enables us to establish a stronger result, in the context of reference-dependent behavior. We next show that this is sufficient to guarantee that the book of complete preorders $\left\{\succeq_{s}\right\}_{s \in X^{*}}$ that rationalizes $c$ satisfies WEA. However, although the extension of the result seems natural, the proof of the equivalence is not 
straightforward.

Theorem 4.8. For any choice correspondence $c$ on $\mathcal{C}(X)$, the following two statements are equivalent:

- $c$ satisfies properties $\alpha, \beta$ and NSC.

- There exists a book $\left\{\succeq_{s}\right\}_{s \in X^{*}}$ of complete preorder relations that rationalizes $c$, and that satisfies WEA.

Note that WEA has bite only in the relevant part of a book, namely, above the reference points. Thus, every book rationalizing $c$ when $\alpha, \beta$ and NSC hold, also satisfies WEA.

Other properties in the literature deal with stronger notions. It is immediate that Sugden's Strictly Exchange-Aversion (SEA), ${ }^{7}$ or Sagi's No-regret (NR), ${ }^{8}$ imply WEA and SQ. Therefore, the following corollary follows immediately from Theorem 4.8.

Corollary 4.9. If there exists a book $\left\{\succeq_{s}\right\}_{s \in X^{*}}$ of complete preorders that rationalizes $c$ and satisfies SEA or NR, then c satisfies properties $\alpha, \beta$ and NSC.

4.3. Independence of the Initial Reference Point. We will say that behavior is independent of the initial reference point if, for any given $T$ in $\mathcal{X}$, "final" choices do not depend on an initial reference point. Consider example 4.3 above. There, the decision-maker exhibits rationalizable and non-dominated behavior but, when confronted with choice problem $(X, x)$ selects $x$, and when presented with choice problem $(X, y)$ chooses $y$. That is, given the choice set, her choice depends on whichever reference point prevails. ${ }^{9}$

Clearly, independence from the initial reference point imposes a great deal of consistency between the preference orders of different reference points. This leads naturally to the following question: Is it possible that reference-dependent behavior that is rationalizable, non-dominated, and independent of the initial reference point may still depend on reference points, or does it instead collapse into a unique order as in the classical setup? We will see that behavior may in fact still depend on reference points. This shows that behavior exhibiting a great deal of rationality may still show reference point dependency.

The following definition states precisely what we mean by "final" choices.

Definition 4.10. Set $L, L \neq \emptyset$, is the limit set of problem $\left(T, s_{0}\right) \in \mathcal{C}(X)$ if the following two properties are satisfied:

\footnotetext{
${ }^{7} \mathrm{SEA}:$ For any $s_{0}, s_{1}, \ldots, s_{n} \in X$ such that $s_{n} \succeq_{s_{n-1}} s_{n-1} \succeq_{s_{n-2}} s_{n-2} \ldots s_{1} \succeq_{s_{0}} s_{0}, s_{n} \succ_{s_{n}} s_{0}$. Munro and Sudgen's (2002) Weak Acyclicity is equivalent to WEA.

${ }^{8} \mathrm{NR}$ : For any $x, y \in X, x \succ_{y}\left(\succeq_{y}\right) y \Rightarrow x \succ_{s}\left(\succeq_{s}\right) y$ for all $s \in X$.

${ }^{9}$ It is interesting to note that given that the behavior of a subject is initial reference point dependent, she can be manipulated in such a way as to increase her well-being (see footnote 14).
} 
- For any RD-chain $\left(T, s_{i}\right)_{i=0}^{n}$ containing a cyclical chain $\left(T, s_{i}\right)_{i=j}^{n}, j \in\{0,1, \ldots, n\}$, it is $\left\{s_{j}, s_{j+1}, \ldots, s_{n}\right\} \subseteq L$.

- For any $z \in L$, there exists an RD-chain $\left(T, s_{i}\right)_{i=0}^{n}$ containing a cyclical chain $\left(T, s_{i}\right)_{i=j}^{n}$ and $z \in\left\{s_{j}, s_{j+1}, \ldots, s_{n}\right\}$.

The limit set of $\left(T, s_{0}\right)$ comprises all the elements that are part of at least one cyclical RD-chain that may arise in an RD-chain starting at $\left(T, s_{0}\right)$. Then, for every choice problem $\left(T, s_{0}\right)$ if a limit set exists, it is always unique. Consider the following example.

Example 4.11. Let $X=\{x, y, z\}, c(X, x)=c(X, y)=c(\{x, y\}, x)=c(\{x, y\}, y)=$ $\{x, y\}, c(X, z)=c(\{y, z\}, y)=c(\{y, z\}, z)=\{y\}, c(\{x, z\}, x)=c(\{x, z\}, z)=\{x\}$, and for any other $(T, s) \in \mathcal{C}(X)$ let $c(T, s)=T$.

Starting from $(X, z)$, the following RD-chains are possible:

(1) $(X, z),(X, y),(X, y), \ldots,(X, y), \ldots$

(2) $(X, z),(X, y),(X, x),(X, y), \ldots,(X, x),(X, y), \ldots$

(3) $(X, z),(X, y),(X, x),(X, x), \ldots,(X, x), \ldots$

That is, the decision-maker may continue cyclically to choose alternative $y$ when the reference point is $y$, or repeatedly switch back and forth between $x$ and $y$, or stick with $x$. Clearly, no cyclical chain starting from $(X, z)$ goes through $z$. Therefore, the limit set of $(X, z)$ is $\{x, y\}$. Hence, the limit set $L$ of a choice problem $(T, s)$ can be regarded as the set of possible final choices.

In Example 4.4, for any $y \in X$ there exists no limit set of $(X, y)$. This is because there exists no cyclical chain. The decision-maker in Example 4.4 locally maximizes the consumption of a good, increasing its consumption level choice by choice. In Example 4.2 the limit set of $(X, x)$ and $(X, y)$ is $\{x, y\}$, and in Example 4.3 the limit sets of $(X, x)$ and $(X, y)$ are $\{x\}$ and $\{y\}$, respectively.

Note that NSC implies that any set of alternatives $V$ that belongs to a limit set $L$ and that constitutes a cyclical chain must be non-strict, or put differently, every change of mind about the preferred alternative involves an indifference comparison. This, however, is not equivalent to the fact that all alternatives in $V$ are indifferent from any reference point in $V$.

The next axiom formally states independence from the initial reference point on choice correspondences.

Limit Set Uniqueness (LSU): For any $T \in \mathcal{X}$ and for any $x, y \in T$, if $L(T, x)$ and $L(T, y)$ exist, then $L(T, x)=L(T, y)$.

On the reference-dependent preferences side, we introduce the following condition, which constitutes a direct restriction on the difference between the orders of distinct 
reference points.

Inverse Status Quo Positivity (ISQP): For any $x, y \in X, x \succ_{x} y$ implies $x \succ_{y} y$.

ISQP goes in the opposite direction to the axioms typically imposed in the study of phenomena related to the status quo bias (see section 5.1). It states that if $x$ is regarded as unambiguously superior to $y$ when $x$ is the reference point, this relation should be maintained when the reference point is $y$, instead. We can now present our next result.

Theorem 4.12. For any choice correspondence $c$ on $\mathcal{C}(X)$, the following two statements are equivalent:

- c satisfies properties $\alpha, \beta, N S C$ and $L S U$.

- There exists a book $\left\{\succeq_{s}\right\}_{s \in X^{*}}$ of complete preorder relations that rationalizes $c$, and that satisfies WEA and ISQP.

To illustrate Theorem 4.4 consider example 4.3. It is easy to see that it satisfies properties $\alpha, \beta$ and NSC, but is not independent of the initial reference point. Example 4.11 describes rationalizable and non-dominated behavior and is at the same time independent of the initial reference point. Further, since $\succeq_{x}, \succeq_{z}$, and $\succeq_{\diamond}$ are mutually different, example 4.11 shows that while independence from the initial reference point, together with rationalizable and non-dominated behavior, imposes a great deal of structure on reference-dependent behavior, there is still room for some degree of reference point influence.

4.4. Path-Independence. So far we have studied the independence of final choices from initial reference points given a set $T \in \mathcal{X}$, but what happens when the choice set can also vary? That is, it is conceivable that a subject with rationalizable and non-dominated behavior also satisfies independence of initial reference points, but her final choices may still depend on the particular sequence of presentation of the choice problems.

The following condition is inspired from Plott's (1973) well-known independence condition.

Plott's Path Independence (PPI): For any sets $T, V \in \mathcal{X}$, for every $s \in T \cap V$ and $z \in L(T, s) \cup L(V, s), L(T \cup V, z)=L(L(T, s) \cup L(V, s), z)$.

PPI states that the limit set of a choice set $T \cup V$, given a particular starting reference point $z$, depends neither on the starting point $z$, nor on a particular decomposition of the choice set. When LSU holds, PPI reduces to $L(T \cup V)=L(L(T) \cup L(V))$. The following example shows, however, that LSU is not a necessary condition for PPI. 
Example 4.13. Let $X=\{x, y, z\}, c(X, x)=c(\{x, y\}, x)=c(\{x, y\}, y)=c(\{x, z\}, x)=$ $c(\{x, z\}, z)=\{x\}, c(X, y)=\{x, z\}, c(X, z)=c(\{y, z\}, z)=\{y\}, c(\{y, z\}, y)=\{z\}$, and for any other $(T, s) \in \mathcal{C}(X)$ let $c(T, s)=T$.

Clearly, $L(X, x) \neq L(X, y)$. Now, it is not difficult to check that PPI holds.

We will now see that LSU is, in combination with NSC, a sufficient condition for PPI. Further, Weak-NSC, together with PPI, imply NSC:

Theorem 4.14. For any choice correspondence $c$ on $\mathcal{C}(X)$ that satisfies properties $\alpha$ and $\beta$ :

- If c satisfies $N S C$ and LSU, then c satisfies PPI.

- If c satisfies Weak-NSC and PPI, then c satisfies NSC.

Hence, an immediate corollary to Theorems and is:

Corollary 4.15. For any choice correspondence $c$ on $\mathcal{C}(X)$, the following two statements are equivalent:

- c satisfies properties $\alpha, \beta$, Weak-NSC, LSU, and PPI.

- There exists a book $\left\{\succeq_{s}\right\}_{s \in X^{*}}$ of complete preorder relations that rationalizes $c$, and that satisfies WEA and ISQP.

Behavior in example 4.11 is rationalizable, non-dominated, and satisfies independence of initial reference points. Then, Theorem 4.4 guarantees that PPI holds here also. Hence, independence from initial reference points, on top of behavior that is rationalizable and non-dominated, is quite a strong condition to impose on referencedependent behavior. It not only requires that, in a given choice set $T$, reference points do not influence final choices, but also implies that the way in which the choice set is presented is non-essential.

Note that since we are not imposing any extra property for path-independence, our conclusion for example 4.11 remains valid. That is, behavior that is rationalizable, non-dominated, independent of the initial reference point, and path-independent does not necessarily collapse into a unique ordering; reference points still matter. We should note here, however, that, with all this structure, and in particular given the result in Theorem 4.4, reference points may still be behaviorally relevant only for the initial choices in an RD-chain.

\section{Applications}

5.1. Status quo bias. There is a large and still-growing experimental and field literature supporting the view that a decision-maker typically values an alternative more highly when it is regarded as the status quo, than otherwise. Versions of this are 
called status quo bias, or endowment effect. ${ }^{10}$ An illuminating experiment that nicely illustrates this bias is the following one, due to Knetsch (1989). A number of participants were randomly split into three groups; call them group $C$, group $M$, and group $N$. Those in group $C$ were given a candy bar, those in group $M$ received a mug, and those in group $N$ got nothing. Then, participants in groups $C$ and $M$ were given the opportunity to change at zero cost their original object for the other. They simply had to express the desire to change the object, and the experimenter would immediately satisfy it. Participants in group $N$ were simply given the opportunity to choose between a candy bar and a mug. The results are surprising. Preferences of subjects in group $N$ were more or less evenly divided, while the great majority in groups $C$ and $M$ expressed no desire to change (90\% of participants in both $C$ and $M)$, showing a strong status quo bias.

The many different versions of the above experiment that have been run have yielded similar results, which suggests a high level of robustness in this finding. The implications of the status quo bias are far more than anecdotal. This bias implies a significant discrepancy in willingness to pay and willingness to accept, as exemplified in the experiments of Kahneman, Knetsch, and Thaler (1990) and Bateman, Munro, Rhodes, Starmer, and Sugden (1997). It also implies a difference in the evaluation of opportunity costs, as opposed to costs of any other nature (see Thaler, 1980). Furthermore, the status quo bias implies that property rights influence the valuation of an object, and hence question the so-called Coase Theorem (see Kahneman, Knetsch, and Thaler, 1990). ${ }^{11}$

In this section we apply the theoretical framework presented earlier to model the status quo bias. In particular, we will show that a choice correspondence that represents the status quo bias is rationalizable by a book of complete preorders that is consistent with non-dominated behavior, but not necessarily independent of initial reference points, or path-independent.

In a recent paper, Masatlioglu and Ok (2005) nicely characterize a choice correspondence representing the status quo bias. Here, we will derive on the book of binary relations the set of axioms that are equivalent to theirs. For the sake of the completeness of the exposition, we introduce Masatlioglu and Ok's (2005) axioms below. For their motivation, however, we refer to their paper.

\footnotetext{
${ }^{10}$ Thaler (1980) was the first to report this phenomenon. Other important experimental and field studies on the subject include Knetsch and Sinden (1984), Samuelson and Zeckhauser (1988), Knetsch (1989), Tversky, Slovic, and Kahneman (1990), Kahneman, Knetsch, and Thaler (1990, 1991), and Bateman, Munro, Rhodes, Starmer, and Sugden (1997). For reviews see Camerer (1995), and Rabin (1998).

${ }^{11}$ One wonders whether "presumption of innocence", which is common practice in the legal systems of many countries, is an implicit recognition of the role of the status quo bias in judgment. According to the status quo bias, the practice of the "presumption of innocence" would reduce the risk of convicting an innocent person, which is typically regarded as a worse scenario than acquitting someone who is guilty.
} 
Dominance (D): For any $(T, s) \in \mathcal{C}(X)$, if $\{y\}=c(T, s)$ for some $T \subseteq V$, and $y \in c(V, \diamond)$, then $y \in c(V, s)$.

Status-quo Irrelevance (SQI): For any $(T, s) \in \mathcal{C}_{\mathrm{rd}}(X)$, if $y \in c(T, s)$ and there is no non-empty $V \subseteq T$ with $V \neq\{s\}$ and $s \in c(V, s)$, then $y \in c(T, \diamond)$.

Status-quo Bias (SQB): For any $(T, s) \in \mathcal{C}(X)$, if $y \in c(T, s)$, then $\{y\}=c(T, y)$.

We now introduce the set of axioms needed to reproduce Masatlioglu and Ok's (2005) results on the book of binary relations. We start with an independence axiom.

Upper Independence (UI): For any $x, y \in X \backslash\{s\}$, such that $x \succ_{s} s$ and $y \succ_{s} s$, $x \succeq_{\diamond} y$ if and only if $x \succeq_{s} y$.

This axiom restricts the influence of the reference point when evaluating pairs of alternatives. If $x$ is evaluated to be at least as good as $y$ when there is no reference point, then for a reference point $s$ such that $x$ and $y$ are strictly preferred to $s, x$ cannot deteriorate relative to $y$. Note, however, that the restrictions imposed by UI are limited. UI restricts the ordering of pairs of alternatives $x$ and $y$ when the qualifier " $x \succ_{s} s$ and $y \succ_{s} s$ " holds.

Now consider the following two axioms.

Status-quo Positivity (SQP): For any $s \in X$ and $y \in X \backslash\{s\}$, if $s \succeq \diamond y$ then $s \succ_{s} y$.

Status-quo Positivity 2 (SQP2): For any distinct $x, y, s \in X$, if $x \succ_{s} s \succ_{s} y$, then $x \succ_{x} y$.

These properties are easily justified by the empirical regularity that we attempt to model here. Property SQP establishes that if, in the absence of a reference point, alternative $s$ is valued to be as at least as good as $y$, when $s$ happens to be the reference point its valuation with respect to $y$ cannot only not deteriorate, it can only improve. Property SQP2 extends the influence of the status quo bias to some cases when there is a reference point. That is, if $x$ is preferred to $y$ from the perspective of the reference point $s$ only when the reference point $s$ is in between $x$ and $y$, SQP2 states that the evaluation of $x$ cannot deteriorate with respect to $y$ when $x$ is itself the reference point. We now introduce the final axiom we need.

Status-quo Singularity (SQS): For any $s, y \in X$, if $s \sim_{s} y$, then $s=y$.

Property SQS strengthens the role of the status quo bias. It is a kind of antisymmetry that applies only to comparisons with the reference point. It imposes either that $y$ is strictly preferred to the reference point $s$, or vice versa. SQS guarantees that, whenever the reference point is chosen, it is uniquely chosen. We can now present 
the following result.

Theorem 5.1. For any choice correspondence $c$ on $\mathcal{C}(X)$, the following two statements are equivalent:

- c satisfies properties $\alpha$ and $\beta$, and axioms $D, S Q I$, and $S Q B$.

- There exists a book $\left\{\succeq_{s}\right\}_{s \in X^{*}}$ of complete preorder relations that rationalizes $c$, and that satisfies UI, SQP, SQP2, and $S Q S$.

Theorem 5.1, together with Masatlioglu and Ok's (2005) Lemma 1 and Theorem 1 immediately imply the following corollary. ${ }^{12}$

Corollary 5.2. For any choice correspondence $c$ on $\mathcal{C}(X)$, the first statement implies the second:

- There exists a book $\left\{\succeq_{s}\right\}_{s \in X^{*}}$ of complete preorder relations that rationalizes $c$, and that satisfies UI, SQP, SQPQ, and $S Q S$.

- There exist $\succeq \subseteq \succeq^{*}$, where $\succeq$ is a partial order and $\succeq^{*}$ is a completion of $\succeq$, such that $c(\cdot, \diamond)=M\left(\cdot \succeq^{*}\right)$, and for all $(T, s) \in \mathcal{C}_{\mathrm{rd}}(X)$

$$
c(T, s)= \begin{cases}\{s\} & \text { if } s \in M(T, \succeq) \\ M\left(T \cap U_{\succ}(s), \succeq^{*}\right) & \text { otherwise }\end{cases}
$$

Furthermore, if $X$ is a non-empty finite set then the above two statements are equivalent.

For a detailed interpretation of the type of choice correspondence obtained in Corollary 5.3 we refer to Masatlioglu and Ok (2005). Here it is sufficient to note that when there is no reference point, the decision-maker selects an element from the set of maximal elements based on a complete preorder relation $\succeq^{*}$. Clearly, this is no more than the standard case and hence $\succeq^{*}=\succeq_{\diamond}$.

When there is a reference point $s$, the decision-maker will only choose $s$ if it belongs to the set of maximal elements of a partial order $\succeq$, indicating a status quo bias. Inspecting the proof of Masatlioglu and Ok's (2005) Lemma 1 we can establish that the partial order $\succeq$ is such that $x \succeq y$ if and only if $x \succeq_{y} y$. Theorem 5.1 clearly shows that any "status quo bias-choice correspondence" is rationalizable by a book of preorder relations. Interestingly, then, the pair of binary relations $\left\{\succeq^{*}, \succeq\right\}$ in Masatlioglu and Ok's result can be directly related to the book of reference-dependent preferences we are working with.

It is easy to show that behavior subject to the status quo bias is non-dominated. By way of contradiction and using NSC, let $\left(T, s_{i}\right)_{i=0}^{n}$ be a cyclical and strict RDchain. Since it is cyclical it must be that $s_{0} \in c\left(T, s_{n}\right)$, but then SQB implies that $\left\{s_{0}\right\}=c\left(T, s_{0}\right)$, which, since $\left(T, s_{i}\right)_{i=0}^{n}$ is strict, constitutes a contradiction.

A choice correspondence representing the status quo bias need not be independent of the initial reference point. The following example represents the case of a "strong"

\footnotetext{
${ }^{12}$ For any $T \in \mathcal{X}, M(T, \succeq)=\{s \in T: y \succ s$ for no $y \in T\}$, and for any $s \in X, U_{\succ}(s)=\{y \in X:$ $y \succ s\}$.
} 
status quo bias that does not satisfy independence of the initial reference point. It can be argued that the example is an abstract representation of Knetsch's experiment. Let $X=\{x, y\}$, where $x \succ_{x} y, y \succ_{y} x$, and $x \sim_{\diamond} y$. When there is no reference point, both alternatives are evaluated by the decision-maker as indifferent, but as soon as the decision-maker has a reference point, she unambiguously prefers the reference point to the other alternative. However, independence of the initial reference point is clearly violated; the final choice depends on which reference point is taken first.

Hence, a decision-maker that manifests the status quo bias nevertheless exhibits choice behavior that can be rationalized through a book of complete preorder relations, and is non-dominated. This suggests that there is still a great deal of rationality in those who are subject to this bias. ${ }^{13}$ However, such a decision-maker could easily be manipulated in situations where an external agent may exercise some influence over the prevalence of a specific reference point, or over the way the choice problem is presented. In this sense, the final choice depends on the discretion of the agent. ${ }^{14}$

It is interesting to explore the structure of status quo choice correspondences when independence from the initial reference point is imposed. To this end, we study the consequences of adding ISQP to the set of properties on reference-dependent preferences we imposed above. Consider SQP again, and note that it implies that, when $x \succ_{y} y$, then $\neg\left(y \succ_{\diamond} x\right)$. By completeness it must be that $x \succeq_{\diamond} y$, and, again by applying SQP, we obtain that $x \succ_{x} y$. Hence, SQP together with completeness implies that if $x \succ_{y} y$, then $x \succ_{x} y$. Remarkably, ISQP involves precisely the reverse implication, namely if $x \succ_{x} y$, then $x \succ_{y} y$. Therefore, if we impose both, ISQP and SQP, we get that for any $x, y \in X, x \succ_{y} y \Leftrightarrow x \succ_{x} y$. Clearly, this imposes a great deal of structure into the book of preference relations. In fact, it is easy to see that when ISQP is added, then $\succeq$ is complete and then it must be that $\succeq=\succeq^{*}=\succeq_{\diamond}$. Consider, by way of contradiction, that there are $x, y \in X$ such that $\neg(x \succeq y)$ and $\neg(y \succeq x)$. Since $x \succeq y$ if, and only if, $x \succeq_{y} y$, completeness of $\succeq_{s}$ implies that $x \succ_{x} y$ and $y \succ_{y} x$, which is a contradiction. Thus, the above discussion leads immediately to the next corollary.

Corollary 5.3. For any choice correspondence $c$ on $\mathcal{C}(X)$, the first statement implies the second:

- There exists a book $\left\{\succeq_{s}\right\}_{s \in X^{*}}$ of complete preorder relations that rationalizes $c$, and that satisfies UI, SQP, SQP2, SQS, and ISQP.

- For all $(T, s) \in \mathcal{C}(X), c(\cdot, s)=M\left(\cdot, \succeq_{\diamond}\right)$.

Furthermore, if $X$ is a non-empty finite set then the above two statements are equivalent.

\footnotetext{
${ }^{13}$ Interestingly, Huck, Kirchsteiger, and Oechssler (2005) theoretically show that decision-makers that exhibit a status quo bias are favored by evolution.

${ }^{14}$ Camerer, Issaharoff, Loewenstein, O'Donoghue, and Rabin (2003) argue that the status quo bias constitutes a perfect example for the possibility of what they call "paternalistic regulation". That is, regulation that could greatly benefit those who are subject to this bias, while causing little harm to those who are not.
} 
That is, if status quo choice behavior is independent of the initial reference point, then this implies that only one preference relation is required to rationalize behavior, $\succeq_{\diamond}$

5.2. Addiction. Our second application concerns addictive behavior and habit formation models. Since Duesenberry (1949), Pollak (1970), and Ryder and Heal (1973) the literature concerning models of habit formation and addiction has attracted the attention of economists. The former is used in variants of growth models to explore life-cycle consumption plans, and is being applied to a wide variety of cases (see, e.g., Campbell 1999). With regard to the latter, the work of Becker and Murphy (1988) has generated an enlightening discussion on how to approach the modelling of addictive behavior (see, e.g., Herrnstein and Prelec 1992, and Elster 1999). The modelling approaches to both types of phenomena are very similar. It is typically assumed that an increase in past consumption increases present and future consumption, and hence the focus is mainly on increasing consumption patterns.

To be more specific, we will interpret the behavior arising from the axioms below on the grounds of addiction, and leave aside the habit formation interpretation. The following describes our notion of addictive behavior.

(1) Dependence on past consumptions: the current reference point is the last level of consumption;

(2) Tolerance: given the actual reference point, the individual either maintains the current level of consumption or increases it;

(3) Saturation: there may be (but not necessarily) a level of consumption over which increments in consumption are not positively evaluated;

(4) Craving: the higher the reference point, the higher the saturation level, if such a level exists; and

(5) Convexity: for any reference point, every alternative between two selected alternatives is also selected.

In line with the approach taken in this paper, (1) assumes a particular type of dependence: the last consumption level determines the current level of addiction. (2) captures the fact that addicts get used to the substance, thus requiring increasingly higher doses. (3) suggests limits to the development of tolerance. (4) captures the fact that the higher the current level of addiction the higher the limit to which the addict is willing to consume. Finally, (5) establishes a sort of continuity in the consumption levels.

We now turn to the characterization we propose. We begin by introducing a linearly ordered universal set of alternatives $(X, \leq)$. That is, $\leq$ is a transitive, complete and antisymmetric binary relation over the non-empty alternative space $X$, and $<$ is the asymmetric part of $\leq$. By way of illustration, consider that $X=[0, k]$ represents the feasible set of grams of some addictive substance, where a natural linear order arises.

The following three properties on choice behavior formally define addictive behavior. 
Monotonicity (M): For any $(T, s) \in \mathcal{C}(X)$, if $y \in c(T, s)$ then $s \leq y$.

This axiom simply states that addictive behavior leads to consumption levels above the reference point, where 'above' is interpreted according to $\leq$. In a recent paper Bossert and Sprumont (2006) formulated a similar property, called Non-deteriorating Choice. Their interpretation is that $\leq$ is a preference relation, and hence, the agent responds by choosing a better alternative than the reference point. Here, however, since we are describing addiction patterns, we maintain a more general interpretation of $\leq$.

Saturation Monotonicity (SM): For any pair $(T, s),(T, r) \in \mathcal{C}(X)$, if $s \leq r$ then, for all $p \in c(T, r)$ there exists $q \in c(T, s)$ such that $q \leq p$.

SM imposes a weak property on the monotonicity of the sets of possible consumption levels selected. It states that if $p$ is chosen from $r$, then from the perspective of any reference point below $r$, say $s$, the set of possible choices should start before $p$. In other words, the saturation level of $s$ (a consumption level above which increments according to $\leq$ are not evaluated as strictly positive) is lower than that of $r$.

Convexity (C): For all $(T, s) \in \mathcal{C}_{\text {rd }}(X)$, if $V \subseteq T, v \in c(V, s), t \in c(T, s)$, if there is $p \in V$ such that $v \leq p \leq t$ or $t \leq p \leq v$ then $p \in c(V, s)$.

Whenever $T=V$, C simply means that any alternative in between two selected ones should also be selected. If $T \neq V$ and $t \in V$, then if properties $\alpha$ and $\beta$ apply, it must also be that $t \in c(V, s)$. However, it could be the case that $t \notin V$. In such an event, $\mathrm{C}$ imposes that any available level in between $v$ and $t$ should be selected when $V$ is the choice set.

We now introduce a set of axioms over a book of binary relations that characterize addictive behavior.

Left Status-quo Positivity (LSQP): For any $s \in X$ and $y \in X \backslash\{s\}$, if $y<s$ then $s \succ_{s} y$.

Notice the analogy between LSQP and axiom SQP, used in the previous application. If the linear order $\leq$ describes the same ranking as $\succeq_{\diamond}$, both axioms are equivalent. This would be the case if, say, a "clean" person who has not yet consumed any of the addictive substance (e.g. cigarettes) were to consider that "more cigarettes are better than less". However, it is perfectly possible for preferences without a reference point $\succeq_{\diamond}$ to be very different. In such cases, both axioms diverge. LSQP establishes that the evaluation of the reference point cannot deteriorate for those initiating in addictive behavior. 
Status-quo Monotonicity (SQM): For any $x \leq y \leq u \leq v$ elements of $X$, such that $v \succ_{x} u \succeq_{x} x$ it must be that $v \succ_{y} u$.

Suppose that the greater element $v$ is clearly preferred to $u$ whenever the reference point is $x$, and both are significant with respect to $x$. If we move the reference point nearer to the pair $u, v$, this comparison must necessarily remain the same.

Absence of Jumps (AJ): If $y \succeq_{x} x, x \leq h \leq y$ then $h \succeq_{x} x$.

If an alternative is preferred to the reference point, intermediate levels should also be preferred to the reference point.

Weak single peakedness (WSP): If $y \succ_{x} z \succeq_{x} x, y \leq z \leq h$ implies $z \succeq_{x} h$.

WSP establishes that, once an agent has chosen to ignore improvements over $\leq$, this pattern is maintained. Notice that standard single peaked preferences satisfy stronger versions of this property. First of all, with single peaked preferences the above restriction is imposed over the entire set $X$, while we only impose it for elements above the reference point. Secondly, single peakedness would require a strict preference whenever $z<h$, which is not our case.

Theorem 5.4. For any choice correspondence $c$ on $\mathcal{C}(X)$, the following two statements are equivalent:

- c satisfies properties $\alpha$ and $\beta$, and axioms $M, S M$ and $C$.

- There exists a book $\left\{\succeq_{s}\right\}_{s \in X^{*}}$ of complete preorder relations that rationalizes $c$, and that satisfies $L S Q P, S Q M, A J$ and WSP.

Addictive behavior is obviously a type of non-dominated behavior. By M, any RD-chain $\left(T, s_{i}\right)_{i=0}^{n}$ must satisfy $s_{i} \leq s_{i+1}, i=0,1, \ldots, n-1$. In order to be strict, it must be that $s_{k}<s_{k+1}$ for some $k$, and therefore, it cannot be cyclical. On the other hand, the following example shows that it is neither necessarily independent of initial reference points, nor, therefore, path-independent.

Example 5.5. Let $X=\{x, y, z\}, x \leq y \leq z, y \succ_{x} x \succ_{x} z, z \succ_{y} y \succ_{y} x$, and $z \succ_{z} y \succ_{z} x$.

If we let $T=\{x, z\}$, then it is clear that LSU does not hold. But the above example says more. It describes a gradual pattern of addiction, and shows that if the choice set could be manipulated, the addiction level of the individual could be kept to a minimum. That is, if the agent starts with a reference point $x$ (e.g., $x=0$ grams of cocaine) and the choice set is $X$, then he will end up consuming the maximum feasible quantity $z$, whereas if he is confronted with choice set $T$, from the perspective of $x$ 
he may find $z$ too much and will then keep "clean". This is an illustration of why educational initiatives emphasizing "all or nothing" consumption patterns may be successful in preventing addiction (for a discussion see Herrnstein and Prelec 1992).

\section{Conclusion}

This paper is a reaction to the accumulated empirical evidence suggesting that behavior is reference-dependent in a variety of environments. It has been our aim to provide an axiomatic characterization of such behavior, general enough to be applicable to the modelling of a wide range of specific phenomena. We believe that the incorporation into economic theory of such well-established and predictable phenomena is a natural step that will eventually help towards the better understanding of economic behavior.

We have studied choice behavior and preference relations, and have gradually imposed rationality demands on them. We have shown that reference-dependent behavior may satisfy a large number of rationality demands, while still being dependent on reference points.

We have axiomatized reference-dependent behavior when the reference point, if it exists, belongs to the choice set. We chose this as the best approach to certain problems, such as those we have modelled here: status quo bias and addiction. In some situations, of course, it may be more appropriate not to impose that the reference point be part of the choice set. Rubinstein and Zhou (1999) constitute an example of the latter. They characterize choice functions that select the closest point to the reference point in the choice set (they axiomatize a "minimal distance" choice function). This suggests what we believe to be a promising line for future research, namely, the characterization of reference-dependent behavior for phenomena where the reference point may not belong to the choice set. ${ }^{15}$

We further suggest that the application of our work may prove fruitful for the modelling of other reference-dependent phenomena, thus opening up another line for future research.

\section{PROOFS}

Proof of Lemma 4.5. Let us first show that when $c$ satisfies axiom WARP, then $c$ satisfies properties $\alpha$ and $\beta$. Consider a choice correspondence satisfying WARP. Suppose, by way of contradiction that $\alpha$ is not satisfied. In this case, there exists a pair of sets $V \subset T$ and a pair of elements $x \in V, s \in V$ or $s=\diamond$ such that $x \in c(T, s)$ but $x \notin c(V, s)$. Since $c$ is non-empty valued by definition, there exists $y \in c(V, s)$, $y \in V \subset T$, thus contradicting WARP. Property $\beta$ is trivial, and therefore, this part of the proof is omitted.

We now show that if $c(T, s)$ satisfies properties $\alpha$ and $\beta$, then there exists a book of transitive relations $\left\{\succeq_{s}\right\}_{s \in X^{*}}$ such that $c(T, s)=\psi\left(T, \succeq_{s}\right)$ and every $\succeq_{s}$ is complete

\footnotetext{
${ }^{15}$ Note however that, as Rubinstein and Zhou state, considering choice problems where the reference point does not necessarily belong to the choice set does not increase the generality of the theory, since the required axioms will be more demanding.
} 
in $X_{s}$. Let $c$ satisfy properties $\alpha$ and $\beta$, and define for all $s$ in $X^{*}$ the binary relation $\succeq_{s}$ on $X$ by $x \succeq_{s} y$ if and only if $x \in c(\{x, y, s\}, s)$. In order to condense the proofs, in the previous definition and throughout the paper, we interpret that whenever $s=\diamond$, $\{x, y, \diamond\}=\{x, y\}$.

We start by checking for transitivity. First note that, whenever $s=\diamond$, we can apply standard results that guarantee the transitivity of $\succeq_{\diamond}$. Then, we have to show that for all $x, y, z, s \in X$, if $x \succeq_{s} y \succeq_{s} z$, then $x \succeq_{s} z$. Let $x \succeq_{s} y \succeq_{s} z$. If $z \in c(\{x, y, z, s\}, s)$, applying property $\alpha$ implies that $z \in c(\{y, z, s\}, s)$, then by property $\beta, y \in c(\{x, y, z, s\}, s)$. Now suppose that $t \in\{y, s\}$ is in $c(\{x, y, z, s\}, s)$, by property $\alpha, t \in c(\{x, t, s\}, s)$. Since, in any case, $x \in c(\{x, t, s\}, s)$, then by property $\beta, x \in c(\{x, y, z, s\}, s)$. Therefore, it must always be that $x \in c(\{x, y, z, s\}, s)$ and by applying property $\alpha$ we get $x \in c(\{x, z, s\}, s)$. This implies $x \succeq_{s} z$ and transitivity, as desired.

We now show that if $y \in c(T, s)$ then $y \in G\left(T, \succeq_{s}\right)$. By property $\alpha, y \in c(T, s)$ implies $y \in c(\{y, h, s\}, s)$ for all $h \in T$, which means $y \succeq_{s} h$ for all $h \in T$. Hence it must be that $y \in G\left(T, \succeq_{s}\right)$. Therefore, $c(T, s) \subseteq G\left(T, \succeq_{s}\right)$, and consequently $G\left(T, \succeq_{s}\right) \neq \emptyset$. Now let $y \in G\left(T, \succeq_{s}\right)$, which implies that $y \succeq_{s} h$ for all $h \in T$. Take any $z \in c(T, s)$. By property $\alpha, z \in c(\{s, y, z\}, s)$. Since $y \succeq_{s} z$, it must also be that $y \in c(\{s, y, z\}, s)$, and hence property $\beta$ guarantees that $y \in c(T, s)$. This concludes the second part of the proof.

We now prove that this book also $M$-rationalizes $c$. Suppose, by way of contradiction, that there exists a set $T$ and an element $s \in X^{*}$ such that $G(T, s) \neq M(T, s)$. Therefore, there must exist an element $x \in M(T, s) \backslash G(T, s)$, which is equivalent to saying that there exists an element $x$ such that $\neg\left(y \succ_{s} x\right)$ for all $y \in T$ and $\neg\left(x \succeq_{s} z\right)$ for at least one $z \in T$. Hence, given the definition of $\succeq_{s}$ the only possibility is that $s=c(\{x, z, s\}, s)$. By $G$-rationalizability, this guarantees that $s \succ_{s} x$, thus contradicting the fact that $x \in M(T, s)$. Therefore the book must also $M$-rationalize c.

We are left to prove that every $\succeq_{s}$ is complete in $X_{s}$. Consider $x, y \in X_{s}$. We have to prove that either $x$ or $y$ is selected in $\{x, y, s\}$. Suppose otherwise. Then, by property $\alpha, s \in c(\{x, y, s\}, s)$ implies that $s \in c(\{x, s\}, s)$. By property $\beta$, it must also be that $x \in c(\{x, y, s\}, s)$, which is an absurd. Therefore, $\succeq_{s}$ is complete in $X_{s}$.

We finally show that if there is a book $\left\{\succeq_{s}\right\}_{s \in X^{*}}$ of transitive relations, such that every $\succeq_{s}$ is complete in $X_{s}$, and that $\psi$-rationalizes $c$, then $c$ satisfies WARP. Standard results apply to the case of $s=\diamond$. Hence, now we study the case where $s \neq \diamond$. Assume by reduction to the absurd that WARP does not hold, i.e., there exists $y \in c(T, s), s, x, y \in T \cap V, x \in c(V, s)$ and $y \notin c(V, s)$. Given that $y \in c(T, s)$, $\psi$-rationalization guarantees that $y \in c(\{y, s\}, s)$ and therefore $y \in X_{s}$. Similarly, given that $x \in c(V, s)$, we can derive that $x \in X_{s}$. Since $y \in c(T, s)=\psi\left(T, \succeq_{s}\right)$, the completeness assumption guarantees that $y \succeq_{s} t$ for every $t \in X_{s} \cap T$. Particularly, $y \succeq_{s} x$. Since $x \in c(V, s)=\psi\left(V, \succeq_{s}\right), x \succeq_{s} v$ for every $v \in X_{s} \cap V$. By transitivity of $\succeq_{s}$, it must also be that $y \succeq_{s} v$ for every $v \in X_{s} \cap V$. Given the completeness assumption on $X_{s}, y \succeq_{s} s$. Now, note that $\psi$-rationalization guarantees that, for every $x \notin X_{s}, s \succ_{s} x$. Therefore, transitivity guarantees that $y \succ_{s} v$ for every 
$v \in\left(X \backslash X_{s}\right) \cap V$. This shows that $y \succeq_{s} v$ for all $v \in V$, which contradicts the original assumption that $c$ is $\psi$-rationalizated. This concludes the argument. Items 1,2 and 3 are equivalent.

The last part of the lemma is straightforward and thus has been omitted.

Proof of Corollary 4.6. The book of preorders $\left\{\succeq_{s}\right\}_{s \in X^{*}}$ used in the proof of the previous lemma can be easily completed. We have proved that $\succeq_{s}$ is complete in $X_{s}$ and that $x \succ_{s} y$ for all $x \in X_{s}$ and $y \in X \backslash X_{s}$. Clearly, $\neg\left(x \succeq_{s} y\right)$ for all $x, y \in X \backslash X_{s}$. Consider then the book of preorders $\left\{\succeq_{s}^{\prime}\right\}_{s \in X^{*}}$ given by $x \succeq_{s}^{\prime} y$ if and only if $\neg\left(y \succ_{s} x\right)$. That is, whenever $x \succeq_{s} y$ we still write $x \succeq_{s}^{\prime} y$ and establish an equivalence relation in $X \backslash X_{s}$. This satisfies the requirements. The rest of the proof follows from Lemma 4.5.

The following lemma will be useful for our next characterization result.

Lemma 7.1. Let $c$ be defined on $\mathcal{C}(X)$ and satisfy properties $\alpha, \beta$, and NSC. Let $\left\{\succeq_{s}\right\}_{s \in X^{*}}$ be a book of complete preorders that rationalizes $c$. Take $s_{n} \succeq_{s_{n-1}} s_{n-1} \succeq_{s_{n-2}}$ $s_{n-2} \ldots s_{1} \succeq_{s_{0}} s_{0}$ with at least one strict inequality. Then, for all $p \in\{1,2, \ldots, n\}$, for all $t \in\{1,2, \ldots, p\}$, we must have $s_{p} \in c\left(\left\{s_{p}, s_{p-1}, \ldots, s_{p-t}\right\}, s_{p-1}\right)$.

Proof of Lemma 7.1. If $t=1$, then, for any $p \in\{1,2, \ldots, n\}$, using $s_{p} \succeq_{s_{p-1}} s_{p-1}$, we conclude that $s_{p} \in c\left(\left\{s_{p}, s_{p-1}\right\}, s_{p-1}\right)$. We will now prove the following induction hypothesis: If the result is valid for any $t=1,2, \ldots, k$ with $k<p$ (otherwise the induction is complete for this value of $p$ ), it is also valid for $t=k+1$. By contradiction, let $s_{p} \notin c\left(\left\{s_{p}, s_{p-1}, \ldots, s_{p-k-1}\right\}, s_{p-1}\right)$. In this case, consider the set $T=$ $\left\{s_{p}, s_{p-1}, s_{p-2}, \ldots, s_{p-k-1}\right\}$. If for some $j \in\{1,2, \ldots, k\}$, we have $s_{p-j} \in c\left(T, s_{p-1}\right)$ then by property $\alpha$, we have $s_{p-j} \in c\left(T \backslash\left\{s_{p-k-1}\right\}, s_{p-1}\right)$ and using the induction hypothesis and property $\beta$, we obtain $s_{p} \in c\left(T, s_{p-1}\right)$, which is absurd. Then, we must have $\left\{s_{p-k-1}\right\}=c\left(T, s_{p-1}\right)$, and by properties $\alpha$ and $\beta\left\{s_{p-k-1}\right\}=c\left(T \backslash\left\{s_{p}\right\}, s_{p-1}\right)$. By hypothesis it must also be that $s_{p-1} \in c\left(T \backslash\left\{s_{p}\right\}, s_{p-2}\right)$. Since $s_{p-k} \succeq_{s_{p-k-1}} s_{p-k-1}$, we can find an element $s_{l_{1}}$ such that $s_{l_{1}} \in c\left(T \backslash\left\{s_{p}\right\}, s_{p-k-1}\right)$ and $l_{1}>p-k-1$. If $l_{1} \neq p-1$ proceed in an analogous way, considering an element $s_{l_{2}}$ such that $s_{l_{2}} \in c\left(T \backslash\left\{s_{p}\right\}, s_{l_{1}}\right)$ and $l_{2}>l_{1}$ (note that such an $l_{2}$ exists because the induction hypothesis is valid for the set $T \backslash\left\{s_{p}\right\}$, smaller than $\left.T\right)$. Since set $T$ is finite, we will find an element $l_{m}=p-1$ such that $s_{l_{m}} \in c\left(T \backslash\left\{s_{p}\right\}, s_{l_{m-1}}\right)$. The sequence of reference-dependent choice problems $\left(T \backslash\left\{s_{p}\right\}, s_{p-1}\right),\left(T \backslash\left\{s_{p}\right\}, s_{p-k-1}\right),\left(T \backslash\left\{s_{p}\right\}, s_{l_{1}}\right), \ldots,\left(T \backslash\left\{s_{p}\right\}, s_{l_{m-1}}\right)$ constitutes a strict RD-chain. However, $s_{p-1}=s_{l_{m}} \in c\left(T \backslash\left\{s_{p}\right\}, s_{l_{m-1}}\right)$, contradicting NSC. Therefore, the induction is valid for $t=k+1$. Thus, the lemma is proved.

Proof of Theorem 4.8. We first show that, given a choice correspondence $c$ satisfying properties $\alpha, \beta$ and NSC, we can find a book of preferences satisfying the requirements. Consider the binary relations $\succeq_{s}$ defined in the proof of Corollary 4.6. They turn out to be not only transitive but complete, and therefore, to conclude this part of the proof we only need to observe that they satisfy WEA. First, consider the 
following property:

Crossed Transitivity (CT): For any collection $s_{0}, s_{1}, \ldots, s_{n} \in X$ such that $s_{n} \succeq_{s_{n-1}} s_{n-1} \succeq_{s_{n-2}} s_{n-2} \ldots s_{1} \succeq_{s_{0}} s_{0}$ with at least one strict inequality, we must have $s_{n} \succ_{s_{n}} s_{0}$.

It is not difficult to show that $\mathrm{CT}$ and WEA are equivalent in our context of complete preorders. Consider a book $\left\{\succeq_{s}\right\}_{s \in X^{*}}$ of complete preorders. To show that WEA implies CT let, by way of contradiction, $s_{0}, s_{1}, \ldots, s_{n} \in X$ such that $s_{n} \succeq_{s_{n-1}} s_{n-1} \succeq_{s_{n-2}} s_{n-2} \ldots s_{1} \succeq_{s_{0}} s_{0}$, with one strict link, say $\succ_{s_{j}}$ with $0 \leq j \leq n-1$, and $s_{n} \sim_{s_{n}} s_{0}$. Then we can write that

$$
s_{j} \succeq_{s_{j-1}} s_{j-1} \succeq_{s_{j-2}} s_{j-2} \cdots s_{0} \succeq_{s_{n}} s_{n} \succeq_{s_{n-1}} s_{n-1} \cdots s_{j+2} \succeq_{s_{j+1}} s_{j+1}
$$

then WEA implies that $s_{j} \succeq_{s_{j}} s_{j+1}$, but this contradicts our original assumption. In an analogous way it can be proved that CT implies WEA.

We proceed to prove that the book defined in the proof of Corollary 4.6 satisfies CT. Let $s_{n} \succeq_{s_{n-1}} s_{n-1} \succeq_{s_{n-2}} s_{n-2} \ldots s_{1} \succeq_{s_{0}} s_{0}$ with at least one strict inequality. Suppose, by way of contradiction, that $s_{0} \succeq_{s_{n}} s_{n}$. Consider the set $V=\left\{s_{0}, s_{1}, \ldots, s_{n}\right\}$. Select a group of elements by means of the following inductive argument:

(1) By hypothesis, there exists an integer $k \in\{0,1, \ldots, n\}$ such that $s_{k} \in c\left(V, s_{n}\right)$. Select the smallest integer in this group and denote it by $p_{1}$.

(2) If $p_{j}=n$, stop the inductive process. Otherwise, to obtain $p_{j+1}$, consider the problem $\left(V, s_{p_{j}}\right)$. Lemma 7.1 guarantees that $s_{p_{j}+1}$ exists in $c\left(\left\{s_{0}, \ldots, s_{p_{j}+1}\right\}, s_{p_{j}}\right)$. Therefore, there exists an integer $k \in\left\{p_{j}+1, \ldots, n\right\}$ such that $s_{k} \in c\left(V, s_{p_{j}}\right)$. Let $p_{j+1}$ denote the smallest integer satisfying this property.

Inductive reasoning gives us a collection of natural numbers $p_{1}, p_{2}, \ldots, p_{m}$ where $m \geq 2$. The sequence of problems $\left(V, s_{n}\right),\left(V, s_{p_{1}}\right), \ldots,\left(V, s_{p_{m-1}}\right)$ constitutes an RDchain, the strictness of which we will now establish. To see this, consider the strict comparison $s_{r+1} \succ_{s_{r}} s_{r}$ with $r \in\{0,1, \ldots, n-1\}$. We need to study two cases:

- $p_{q} \leq r<p_{q+1}$. We will show that $p_{q} \notin c\left(V, p_{q}\right)$. To this end we need to analyze two cases. First, if $p_{q+1}-p_{q}=1$, the preference between these two elements is strict, and then $p_{q} \notin c\left(V, p_{q}\right)$. Second, if the difference is greater, then suppose, by way of contradiction, that $p_{q} \in c\left(V, p_{q}\right)$. In this case, $p_{q}+1$ is also in $c\left(V, p_{q}\right)$, thus contradicting the definition of $p_{q+1}$.

- $p_{1}>r$. In this case, it is easy to see that $s_{n} \notin c\left(V, s_{n}\right)$. Otherwise, $0=p_{1}$, which using $s_{0} \succeq_{s_{n}} s_{n}$ contradicts $p_{1}>r$.

We have proved that the RD-chain is strict. Therefore, by NSC, it must be that $s_{n} \notin c\left(V, s_{p_{m-1}}\right)$, thus contradicting $s_{p_{m}}=s_{n} \in c\left(V, s_{p_{m-1}}\right)$. Hence $s_{0} \succeq_{s_{n}} s_{n}$ cannot hold and, $\succeq_{s_{n}}$ being complete, we obtain $s_{n} \succ_{s_{n}} s_{0}$. This proves that the book of preferences satisfies CT and therefore, WEA.

We now prove that if there is a book of reference-dependent complete preorders satisfying WEA, the corresponding choice mapping satisfies properties $\alpha, \beta$ and NSC. Taking into account Lemma 4.5, we only need to prove NSC. Consider a strict chain $\left(T, s_{i}\right)_{i=0}^{n}$. Since the chain is strict, there must exist $0 \leq k<n$ and $y \in T$ such 
that $y \succ_{s_{k}} s_{k}$. Since $s_{i} \in c\left(T, s_{i-1}\right)$, and given completeness, we must have that $s_{i} \succeq_{s_{i-1}} h, i=1,2, \ldots, n$, for all $h \in T$. In particular, we have that $s_{k+1} \succeq_{s_{k}} y$ and by transitivity, we obtain that $s_{k+1} \succ_{s_{k}} s_{k}$. We therefore obtain a sequence of comparisons

$$
s_{n} \succeq_{s_{n-1}} s_{n-1} \succeq_{s_{n-2}} s_{n-2} \ldots s_{k+1} \succ_{s_{k}} s_{k} \ldots s_{1} \succeq_{s_{0}} s_{0}
$$

and by applying CT we must have that $s_{n} \succ_{s_{n}} s_{0}$, which shows that $s_{0}$ is not in $M\left(T, \succeq_{s_{n}}\right)=c\left(T, s_{n}\right)$, as desired.

Proof of Theorem 4.4. We begin by showing that if $c$ satisfies properties $\alpha, \beta$, NSC and LSU, then there exists a book $\left\{\succeq_{s}\right\}_{s \in X^{*}}$ of complete preorder relations that rationalizes $c$, and that satisfies WEA and ISQP. Take the book of complete preorders defined in the proof of Lemma 4.5. By considering Theorem 4.8, we only need to check ISQP. Consider, by way of contradiction, two elements $x, y \in X$ such that $x \succ_{x} y$ but $y \succeq_{y} x$. Since the book rationalizes $c$, we must have $\{x\}=c(\{x, y\}, x)$ and $y \in c(\{x, y\}, y)$. Thus, the limit set of $(\{x, y\}, x)$ is $\{x\}$, while the limit of $(\{x, y\}, y)$ must contain $y$, thus contradicting LSU.

We now prove the converse statement. Consider Theorem 4.8, then we only need to check LSU. Suppose, by way of contradiction, that there exists a subset $T$ and elements $x, y \in T$ such that

- $L$ is the limit set of $(T, x)$, and

- $L^{\prime}$ is the limit set of $(T, y)$,

with $L \neq L^{\prime}$.

Without loss of generality, suppose that $z \in L$ and $z \notin L^{\prime}$, and take $w \in L^{\prime}$. By NSC, for all $s \in L \cup L^{\prime}$ we must have that $s \in c(T, s)$ (otherwise, the cyclical subchain containing $s$ would be strict, which is absurd). Then, $z \in c(T, z)$ and $w \in c(T, w)$, which implies that $z \succeq_{z} w$ and $w \succeq_{w} z$. By ISQP we must have $z \sim_{z} w$ and $w \sim_{w} z$. Thus, $z \in c(T, w)$ and $w \in c(T, z)$. There exists a cyclical RD-chain $\left(T, s_{i}\right)_{i=0}^{k}$ such that $s_{0}=y$ and $s_{k}=w$. Then, the chain $\left(T, s_{i}\right)_{i=0}^{k+1}$, with $s_{k+1}=z$ implies that $z \in L^{\prime}$, which is absurd.

Proof of Theorem 4.4. To see that NSC is implied by PPI and Weak-NSC consider, by way of contradiction, a cyclical and strict RD-chain where the reference set $T$ contains at least 3 elements. Since the chain is strict, there exists a pair of elements $x, y$ such that $x \notin c(T, x), y \in c(T, x)$. Clearly, $x, y \in L(T, y)$. Properties $\alpha$ and $\beta$ guarantee that $\{y\}=c(\{x, y\}, x)$ and therefore, using Weak-NSC, it is clear that $L(\{x, y\})=\{y\}$. Then, $L(T, y) \neq L(L(\{x, y\}, y) \cup L(T \backslash\{x\}, y), y)$. Therefore, PPI does not hold, which is a contradiction. Finally Weak-NSC concludes the proof.

We now show that LSU and NSC are sufficient to imply PPI. Given LSU, to show that PPI is implied, we only have to show that $L(T \cup V)=L(L(T) \cup L(V))$, for every $T, V \in \mathcal{C}(X)$. Let $T$ and $V$ be any two sets in $\mathcal{C}(X)$. First, let $z \in L(T \cup V)$ and, without loss of generality, let $z \in T$. Then, by NSC, it must be that $z \in c(T \cup V, z)$. Further, by property $\alpha$ we have that $z \in c(T, z)$, and hence by LSU, $z \in L(T)$. 
Hence, we can apply property $\alpha$ again to get $z \in c(L(T) \cup L(V), z)$, and by LSU, $z \in L(L(T) \cup L(V))$. Then we have that $L(T \cup V) \subseteq L(L(T) \cup L(V))$.

Now let $z \in L(L(T) \cup L(V))$. The case where $z \in T$ and $z \in V$ is trivial. So consider, without loss of generality, that $z \in T$ and $z \notin V$. By NSC, $z \succeq_{z} h$ for all $h \in T \cup L(V)$. Let there exist a $t \in V \backslash L(V)$ such that $t \succ_{z} z$. By LSU there are $s_{0}, s_{1}, \ldots, s_{n} \in V$ and $x \in L(V)$ such that $x \succeq_{s_{n}} s_{n} \succeq_{s_{n-1}} s_{n-1} \succeq_{s_{n-2}} s_{n-2} \ldots s_{1} \succeq_{s_{0}}$ $s_{0} \succeq_{t} t \succ_{z} z$. Then, by Theorem 4.4 WEA holds, which implies that $x \succ_{x} z$, and by ISQP we have that $x \succ_{z} z$, a contradiction. Hence, $z \succeq_{z} h$ for all $h \in T \cup V$, and then $L(L(T) \cup L(V)) \subseteq L(T \cup V)$, as desired.

Proof of Theorem 5.1. We first show that if there is a book of complete preorders $\left\{\succeq_{s}\right\}_{s \in X^{*}}$ that satisfies UI, SQP, SQP2, SQS, and $c(T, s)=M\left(T, \succeq_{s}\right)$, then $c$ satisfies properties $\alpha$ and $\beta$, and axioms D, SQI, and SQB. The implication on properties $\alpha$ and $\beta$ is shown in Lemma 4.5 .

- D: Let $y \neq s \neq \diamond$. Let $\{y\}=c(T, s)=M\left(T, \succeq_{s}\right)$, and $y \in c(V, \diamond)=M\left(V, \succeq_{\diamond}\right)$, $T \subseteq V$. Then $y \succ_{s} h$ for all $h \in T \backslash\{y\}$, and $y \succeq_{\diamond} h$ for all $h \in V$. For all $t \in V \backslash\{s, y\}$, either $t \succ_{s} s$ or $s \succeq_{s} t$. When $t \succ_{s} s$, given that $y \succ_{s} s$, then by UI, $y \succeq_{\diamond} t$ implies that $y \succeq_{s} t$. If $s \succeq_{s} t$, by SQS $s \succ_{s} t$. Now, assume, by way of contradiction, that $t \succ_{s} y$. Then, since $y \succ_{s} s$ and $s \succ_{s} t, M\left(\{s, y, t\}, \succeq_{s}\right)=\emptyset$, which is a contradiction. Then it must be that $y \succeq_{s} t$, and therefore, $y \in M\left(V, \succeq_{s}\right)=c(V, s)$. Now let $y=s \neq \diamond$. Then $y \in c(V, \diamond)=M\left(V, \succeq_{\diamond}\right)$ implies that $y \succeq_{\diamond} h$ for all $h \in V$. By SQP $y \succ_{y} h$ for all $h \in V \backslash\{y\}$, and hence $y \in M\left(V, \succeq_{y}\right)=c(V, y)$. Finally, the case when $s=\diamond$ is trivial, and therefore omitted.

- SQI: Let $y \in c(T, s)=M\left(T, \succeq_{s}\right)$, and assume that there exists no non-empty $V \subseteq T$ with $V \neq\{s\}$ and $s \in c(V, s)$. Then $y \succeq_{s} h$ for all $h \in T$, and $h \succ_{s} s$ for all $h \in T \backslash\{s\}$. By applying UI we get $y \succeq_{\diamond} h$ for all $h \in T \backslash\{s\}$. Finally, it must be that $y \succ_{\diamond} s$, since otherwise $s \succeq_{\diamond} y$ implies, by SQP, that $s \succ_{s} y$, a contradiction. Hence, $y \in M\left(T, \succeq_{\diamond}\right)=c(T, \diamond)$.

- SQB: Let $y \neq s \neq \diamond$ and $y \in c(T, s)=M\left(T, \succeq_{s}\right)$. Then, $y \succeq_{s} h$ for all $h \in T$. By SQS, $y \succeq_{s} s$ implies $y \succ_{s} s$. For all $z \in T \backslash\{s, y\}$, either $s \succeq_{s} z$ or $z \succ_{s} s$. If $s \succeq_{s} z$, by SQS $s \succ_{s} z$. Then $y \succ_{s} s \succ_{s} z$, by SQP2, implies $y \succ_{y} z$. If $z \succ_{s} s$, then $y \succeq_{s} z$, by UI, implies $y \succeq_{\diamond} z$, and SQP implies $y \succ_{y} z$. Now, if $s \succ_{\diamond} y$, SQP would imply that $s \succ_{s} y$, a contradiction. Hence, it must be that $y \succ_{\diamond} s$, and then SQP implies that $y \succ_{y} s$. Hence $\{y\}=M\left(T, \succeq_{y}\right)=c(T, y)$. Now let $y \neq s=\diamond$. Then if $y \in c(T, \diamond)=M\left(T, \succeq_{\diamond}\right), y \succeq_{\diamond} h$ for all $h \in T$, by SQP $y \succ_{y} h$ for all $h \in T \backslash\{y\}$, and then $\{y\}=M\left(T, \succeq_{y}\right)=c(T, y)$. Finally, let $y=s \neq \diamond$. If $y \in c(T, y)=M\left(T, \succeq_{y}\right), y \succeq_{y} h$ for all $h \in T$, by SQS $y \succ_{y} h$ for all $h \in T \backslash\{y\}$, and then $\{y\}=M\left(T, \succeq_{y}\right)=c(T, y)$.

To show the reverse implication, we will use the book of binary relations defined in the proof of Lemma 4.5, where it was shown that if $c$ satisfies properties $\alpha$ and $\beta$, then $\left\{\succeq_{s}\right\}_{s \in X^{*}}$ is a book of complete preorders, and that $c(T, s)=M\left(T, \succeq_{s}\right)$.

- UI: Let $x \succ_{s} s, y \succ_{s} s$. We will show both implications for the case of $x \neq y$; the case of $x=y$ is trivial. Let $x \succeq_{s} y$, then by definition, either $x \in c(\{x, y, s\}, s)$ or $\{s\}=c(\{x, y, s\}, s)$. If $\{s\}=c(\{x, y, s\}, s)$, then properties $\alpha$ and $\beta$ imply that $\{s\}=$ 
$c(\{x, s\}, s)$, which contradicts $x \succ_{s} s$. Then, it must be that $x \in c(\{x, y, s\}, s)$. Note that $s \notin c(\{x, s\}, s)$, and $s \notin c(\{y, s\}, s)$. If $s \in c(\{x, y, s\}, s)$ then by property $\alpha$ we get $s \in c(\{x, s\}, s)$, a contradiction. Hence we can apply SQI to get $x \in c(\{x, y, s\}, \diamond)$. By property $\alpha, x \in c(\{x, y\}, \diamond)$, as desired. Let us now assume that $x \succeq_{\diamond} y$, and by way of contradiction, $\neg\left(x \succeq_{s} y\right)$. Then $x \in c(\{x, y\}, \diamond)$, and $\{y\}=c(\{x, y, s\}, s)$. By SQI we get $y \in c(\{x, y, s\}, \diamond)$. By applying properties $\alpha$ and $\beta, x \in c(\{x, y, s\}, \diamond)$. Now, since $\{x\}=c(\{x, s\}, s)$ and $x \in c(\{x, y, s\}, \diamond)$, by D, $x \in c(\{x, y, s\}, s)$, which is a contradiction. Hence it must be that $x \succeq_{s} y$.

- SQP: Let $s \succeq \diamond y$, then it must be that $s \in c(\{s, y\}, \diamond)$. By applying SQB, we get $\{s\}=c(\{s, y\}, s)$, as desired.

- SQP2: Let $x \succ_{s} s \succ_{s} y$, by transitivity, $x \succ_{s} y$, which by definition implies that $x \in c(\{x, y, s\}, s)$. By SQB $\{x\}=c(\{x, y, s\}, x)$, and by applying properties $\alpha$ and $\beta$, we get $\{x\}=c(\{x, y\}, x)$.

- SQS: Let $s \sim_{s} y$. Since choice correspondences are non-empty valued by definition, the case of no comparability between $s$ and $y$ does not apply here. Then $s \in c(\{y, s\}, s)$ and $y \in c(\{y, s\}, s)$. By SQB $\{s\}=c(\{y, s\}, s)$, and hence $y=s$.

Proof of Theorem 5.4. We first show that if $c$ satisfies the mentioned properties, we can find a book of preferences as described. Consider the book of complete preorders that rationalizes $c$ in the proof of Lemma 4.5. If $x>y$, by $\mathrm{M}$, we cannot have $y \in c(\{x, y\}, x)$ and therefore, $x \succ_{x} y$, which proves LSQP.

To show SQM, consider $x \leq y \leq u \leq v$ with $v \succ_{x} u \succeq_{x} x$. First of all, we will prove that $v \succ_{x} y$. Otherwise, $y \succeq_{x} v \succ_{x} u \succeq_{x} x$ and therefore, $y \in c(T, x)$ where $T=\{x, y, u, v\}$. At the same time $v \in c(V, x)$ with $V=\{x, u, v\}$. Since $y \leq u \leq v$ and $u \in V$, by applying $\mathrm{C}$, we must have that $u \in c(V, x)$, which is absurd because $v \succ_{x} u$. Thus, $v \succ_{x} y$. Given the hypothesis, we must have that $\{v\}=c(T, x)$. The application of SM leads to $\{v\}=c(T, y)$ and therefore, $v \succ_{y} u$.

To show AJ, suppose that $y \succeq_{x} x \succ_{x} h$ with $x \leq h \leq y$. Consider the sets $T=\{x, y, h\}$ and $V=\{x, h\}$. By hypothesis, $y \in c(T, x)$ and $\{x\}=c(V, x)$. The application of $\mathrm{C}$ leads to $h \in c(V, x)$. However, this is absurd, since we cannot have $h=x$.

To prove WSP, suppose $y \succ_{x} z \succeq_{x} x$ and $y \leq z \leq h$. By way of contradiction, let us suppose that $h \succ_{x} z$. There are two possibilities:

- If $y \succeq_{x} h$, then consider $T=\{x, y, z, h\}$ and $V=\{x, z, h\}$. It is obvious that $y \in c(T, x)$ while $h \in c(V, x)$. By C it must also be that $z \in c(V, x)$, which is absurd, because $h \succ_{x} z$.

- If $h \succ_{x} y$, consider the sets $T=\{x, y, z, h\}$ and $V=\{x, y, z\}$. Then we have $h \in c(T, x)$ and $y \in c(V, x)$. The application of $\mathrm{C}$ again leads to $z \in c(V, x)$, which is absurd because $y \succ_{x} z$.

This concludes the first part of the proof.

Now suppose that there exists a book satisfying the requirements. Proving $\mathrm{M}$ is straightforward. To prove SM, consider $x \leq y$ and $p \in c(T, y)$. By $\mathrm{M}$, we must have that $x \leq y \leq p$. If $p \in c(T, x)$, the proof is complete. If not, consider any $q \in c(T, x)$ such that $p<q$. In this case, $q \succ_{x} p$ and $q \succeq_{x} x$. AJ guarantees that $p \succeq_{x} x$. Then, 
by applying SQM, we have that $q \succ_{y} p$ and therefore, $p \notin c(T, y)$, which is absurd. Therefore, there are no such elements and any $h \in c(T, x)$ must fulfil $h<p$, which proves SM.

To end the proof, we only need to show that $\mathrm{C}$ is satisfied. Consider $V \subseteq T$, $v \in c(V, x)$ and $t \in c(T, x)$. Let $p \in V$, with $v \leq p \leq t$ and suppose, by way of contradiction, that $p \notin c(V, x)$. In this case, we must have that $v \succ_{x} p$ and $x \leq v \leq p \leq t$, otherwise, by LSQP $x \succ_{x} v$, which is a contradiction. Since $t \succeq_{x} x$, AJ implies that $p \succeq_{x} x$. Therefore, $v \succ_{x} p \succeq_{x} x$ and $v \leq p \leq t$ guarantees $p \succeq_{x} t$ by application of WSP. Therefore $p \in c(T, x)$ and by property $\alpha, p \in c(V, x)$, which is absurd. The case in which $t \leq p \leq v$ is very similar and is, therefore, omitted.

\section{REFERENCES}

[1] Arrow, K. (1959), "Rational Choice Functions and Orderings," Economica, 26:121-127.

[2] Bateman, I., A. Munro, B. Rhodes, C. Starmer, and R. Sugden (1997), "A Test of the Theory of Reference-Dependent Preferences," Quarterly Journal of Economics, 112:479-505.

[3] Becker, G. and K. Murphy (1988), "A Theory of Rational Addiction," Journal of Political Economy, 96:675700.

[4] Bleichrodt, H. (2006), "Prospect Theory with Reference Points in the Opportunity Set," unpublished manuscript, Erasmus University.

[5] Bleichrodt, H. (2007), "Reference-Dependent Utility with Shifting Reference Points and Incomplete Preferences," Journal of Mathematical Psychology 51:266-276.

[6] Bossert, W. and Y. Sprumont (2006), "Non-Deteriorating Choice," unpublished manuscript, Université de Montréal.

[7] Camerer, C. (1995), "Individual Decision Making," in J. Kagel and A. Roth, eds., Handbook of Experimental Economics, Princeton: Princeton University Press.

[8] Camerer, C., S. Issaharoff, G. Loewenstein, T. O'Donoghue, and M. Rabin (2003), "Regulation for Conservatives: Behavioral Economics and the Case for Asymmetric Paternalism," University of Pennsylvania Law Review, 151:1211-1254.

[9] Campbell, J.Y. (1999), "Asset Prices, Consumption, and the Business Cycle," in J.B. Taylor and M. Woodford, eds., Handbook of Macroeconomics, North-Holland.

[10] Danan, E. (2005), "Money Pumps for Incomplete and Discontinuous Preferences," unpublished manuscript, Universite de Cergy-Pontoise.

[11] Duesenberry, J. (1949), Income, Saving, and the Theory of Consumer Behavior, Cambridge: Harvard University Press.

[12] Elster, J. (Ed.) (1999), Addiction: Entries and Exits, New York: Russell Sage Foundation.

[13] Fishburn, P. (1988), Non linear preference and utility theory, Baltimore: Johns Hopkins.

[14] Giraud, R. (2004), "Reference-Dependent Preferences: Rationality, Mechanism and Welfare Implications," unpublished manuscript, Univeristy of Paris I.

[15] Giraud, R. (2006), "Reference-Dependent Preferences: An Axiomatic Approach to the Underlying Cognitive Process," in Cognitive Economics: New trends, eds. B. Walliser and R. Topol, Elsevier.

[16] Giraud, R. (2007), "Money Matters: An Axiomatic Exploration of the Endowment Effect and the Preference Reversal Phenomenon," unpublished manuscript, University of Paris I.

[17] Herrnstein R.J. and D. Prelec (1992), "A Theory of Addiction," in G.F. Loewenstein and J.Elster (eds.) Choice over Time, New York: Russell Sage Foundation.

[18] Houthakker, H.S. (1950), "Revealed Preference and the Utility Function," Economica, 17:159174. 
[19] Huck, S., G. Kirchsteiger, and J. Oechssler (2005), "Learning to Like What You Have: Explaining the Endowment Effect," Economic Journal, 115:689-702.

[20] Kahneman, D. and A. Tversky (1979), "Prospect Theory: An Analysis of Decision Under Risk," Econometrica, 47:263-291.

[21] Kahneman, D., J. Knetsch, and R. Thaler (1990), "Experimental Tests of the Endowment Effect and the Coase Theorem," Journal of Political Economy, 98:1325-1348.

[22] Kahneman, D., J. Knetsch, and R. Thaler (1991), "Anomalies: The Endowment Effect, Loss Aversion, and Status Quo Bias," Journal of Economic Perspectives, 5:193-206.

[23] Köszegi, B. and Rabin, M. (2006), "A Model of Reference-Dependent Preferences", Quarterly Journal of Economics, 121:1133-1165.

[24] Knetsch, J. (1989), "The Endowment Effect and Evidence of Nonreversible Indifference Curves," American Economic Review, 79:1277-1284.

[25] Knetsch, J. and Sinden, J. (1984), "Willingness to Pay and Compensation Demanded: Experimental Evidence of an Unexpected Disparity in Measures of Value," Quarterly Journal of Economics, 99:507-521.

[26] Mandler, M. (2005), "Incomplete Preferences and Rational Intransitivity of Choice," Games and Economic Behavior, 50:255-277.

[27] Masatlioglu, Y. and E.A. Ok (2005), "Rational Choice with Status Quo Bias," Journal of Economic Theory, 121:1-29.

[28] Masatlioglu, Y. and E.A. Ok (2006), "Reference-Dependent Procedural Decision Making," unpublished manuscript, NYU.

[29] Masatlioglu, Y. and N. Uler (2005), "Competing Reference Dependent Models," unpublished manuscript, University of Michigan.

[30] Mas-Colell, A., M.D. Whinston, and J.R. Green (1995), Microeconomic Theory. New York: Oxford University Press.

[31] Munro, A. and Sugden, R. (2003), "On the Theory of Reference-Dependent Preferences," Journal of Economic Behavior and Organization, 50:407-428.

[32] Plott, C.R. (1973), "Path Independence, Rationality, and Social Choice," Econometrica, 41:1075-1091.

[33] Pollak, R.A. (1970), "Habit Formation and Dynamic Demand Functions," Journal of Political Economy, 78:745763.

[34] Raiffa, H. (1968), Decision analysis, MA: Addison-Wesley.

[35] Rabin, M. (1998), "Psychology and Economics," Journal of Economic Literature, 36:11-46.

[36] Rubinstein, A. and L. Zhou (1999), "Choice Problems with a 'Reference' Point," Mathematical Social Sciences, 37:205-209.

[37] Ryder, H. and G. Heal (1973), "Optimal Growth with Intertemporally Dependent Preferences," Review of Economic Studies, 40:133.

[38] Sagi, J. (2006), "Anchored Preference Relations," Journal of Economic Theory, 130:283-295.

[39] Samuelson, W. and R. Zeckhauser (1988), "Status Quo Bias in Decision Making," Journal of Risk and Uncertainty, 1:7-59.

[40] Sen, A. (1969), "Quasi-Transitivity, Rational Choice, and Collective Decisions," Review of Economic Studies, 36: 381-93.

[41] Sugden, R. (2003), "Reference-Dependent Subjective Expected Utility," Journal of Economic Theory, 111:172-191.

[42] Thaler, R. (1980), "Toward a Positive Theory of Consumer Behavior," Journal of Economic Behavior and Organization, 1:39-60.

[43] Tversky, A. and D. Kahneman (1991), "Loss Aversion in Riskless Choice: A reference-dependent Model," Quarterly Journal of Economics, 106:1039-61.

[44] Tversky, A., P. Slovic, and D. Kahneman (1990), "The Causes of Preference Reversal," American Economic Review, 80:204-217. 
[45] Zhou, L. (1997), "Revealed Preferences and Status Quo Effect," unpublished manuscript, Duke University. 